\title{
BMJ Open Population-level impact of diabetes integrated care on commissioner payments for inpatient care among people with type 2 diabetes in Cambridgeshire: a postintervention cohort follow-up study
}

Dahai Yu, ${ }^{1,2}$ Wei Yang, ${ }^{1,3}$ Yamei Cai, ${ }^{1}$ Zhanzheng Zhao, ${ }^{1}$ David Simmons ${ }^{4}$

To cite: Yu D, Yang W, Cai Y, et al. Population-level impact of diabetes integrated care on commissioner payments for inpatient care among people with type 2 diabetes in Cambridgeshire: a postintervention cohort follow-up study. BMJ Open 2017;7:e015816. doi:10.1136/ bmjopen-2017-015816

- Prepublication history and additional material for this paper are available online. To view these files, please visit the journal online (http://dx.doi. org/10.1136/bmjopen-2017015816).

Received 2 January 2017 Revised 28 November 2017 Accepted 29 November 2017

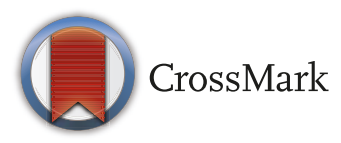

For numbered affiliations see end of article.

Correspondence to Professor Zhanzheng Zhao; zhanzhengzhao@zzu.edu.cn and Professor David Simmons; dsworkster@gmail.com

\section{ABSTRACT}

Objectives Few studies have estimated the effect of diabetes integrated care at a population level. We have assessed the impact of introducing a community serviceled diabetes integrated care programme on commissioner payments (tariff) for inpatient care in rural England. Methods The Diabetes Integrated Care Initiative was delivered by a separate enhanced community diabetes service, increasing specialist nursing, dietetic, podiatry and medical support to primary care and patients, while linking into other diabetes specialist services. Commissioner data were provided by the local authority. The difference in area between the two overlapping distribution curves of inpatient payments at baseline and follow-up (at 3 years) was used to estimate the effect of integrated care on commissioner inpatient payments on a population level. Results Over the 3-year period, reduced inpatient payments occurred in $2.7 \%(1.3 \%$ to $5.8 \%)$ of patients with diabetes aged more than 70 years in the intervention area. However, reduced diabetes inpatient payments occurred in $3.20 \%(1.77 \%$ to $7.20 \%)$ of patients aged $<70$ years and $4.1 \%(2.3 \%$ to $7.9 \%)$ of patients $\geq 70$ years in one of the two adjacent areas.

Conclusion This enhanced community diabetes services was not associated with substantially reduced inpatient payments. Alternative diabetes integrated care approaches (eg, with direct primary and secondary care collaboration rather than with a community service) should be tested.

\section{INTRODUCTION}

As the social and economic impact of diabetes grows, so does the variety of attempts to improve care quality and reduce healthcare costs among those affected. ${ }^{1-4}$ One approach, which is able to provide at least equivalent care to routine medical care with some types of patients, has been the introduction of nurses working within protocols, within medical services. ${ }^{5}$ Other models known as 'intermediate care', including general practitioners
Strengths and limitations of this study

- The 'health gain' in the revised method was clearly defined with a formulated algorithm of evaluation, which broadened the utilisation scenarios especially when negative values were raised.

- The data used in this study depended on the completeness of the coding for diabetes in the general practitioner records. The impact of this potential ascertainment bias should have been steady as no systematic change in coding was known to have occurred over this time period.

- Data on some important confounders such as lipid profiles were not available in this study.

with a special interest, ${ }^{6}$ and community diabetes nursing services ${ }^{6}$ have been implemented, but without robust evaluation. As a proposed system, integrated care articulates all health workers and health systems around the needs of each patient and should be associated with improved outcomes and less cost. ${ }^{7}$

However, the impact of a population-based integrated care intervention is difficult to measure on an individual level. One randomised trial of an intermediate care service achieved minimal actual incremental benefit. ${ }^{8}$ By their nature, randomised controlled trials are difficult to use when assessing the impact of a complete system change at a population level. Sarkadi et al have proposed a method to look at population outcomes in their own right in the quest of understanding how interventions work at a population level. ${ }^{9}$ Under the English National Health Service (NHS), public inpatient care is paid for from taxation through local commissioners. These payments do not generally cover the hospital costs of inpatients 
with diabetes, ${ }^{10}$ but can provide an NHS commissioner perspective that reflects both acuity and complexity, beyond, for example, length of stay. We have now used the Sarkadi approach to assess whether any changes in population-based commissioner inpatient payment data occurred during a diabetes integrated care intervention by viewing the level and distribution of commissioner inpatient payments in the population as the unit of interest.

\section{METHODS}

East Cambridgeshire and Fenland (ECF: 2009 population 160000 , diabetes population 7790) is largely rural, with a small number of socioeconomically deprived communities. ${ }^{6}$ There is no local major hospital (with, eg, an emergency department) falling within the catchment areas of four hospitals outside of the area. Some diabetes outcomes have been historically poor. ${ }^{11}$ A separate, local, diabetes specialist nurse-led community service was introduced in 2003. ${ }^{12}$ From April 2009, this was replaced with a new Diabetes Integrated Care Initiative (DICI) using additional finance ( $£ 250000 \mathrm{pa}$ ), in an attempt to address continuing health disparities. The components of the DICI have been described in the previous publications. ${ }^{13}$ The health district includes two other areas, Huntingdonshire and Greater Cambridge, which did not receive the full intermediate team and are able to serve as 'control' areas, although each hospital-based service would have continued with its own internal service developments. We have previously reported no impact on metabolic control or hospitalisation rates in spite of full implementation of the service. ${ }^{6}$

Deidentified electronic Secondary Uses Service data for across Cambridgeshire were obtained for recorded inpatient tariff between April 2007 (ie, 2 years before the DICI contract commenced) and March 2012. Practice, patient age, elective/non-elective status,International Classification of Diseases 10th revision (ICD10) and Health Related Group coding were included in the dataset. Diabetes was considered present if E10- E14 was in any ICD10 field and, as the primary cause of admission if coded in the first field. ${ }^{14} 15$ Inpatient payments recorded in 2008-2009 were used as baseline, to compare with that recorded in 2011-2012 as the end of the intervention period. Using the Sarkadi et al method, the mean and SD for normal distributions before and after the intervention can be estimated. The 'health gain' is defined as the area between the two distribution curves on the right side, where the distribution density after intervention is lower (the shaded area in online supplementary figure 1 left). In our study, 'health gain' represents the proportion of patients with reduced inpatient payments between the baseline and intervention period. The reduction in commissioner payments reflects reduced needs in care and thus improvement in health.

Sarkadi's method has outlined ways to calculate the impact when the two distributions have the same SD, or when the follow-up group has smaller mean and smaller $\mathrm{SD}$ at the same time. However, we have noticed when using real data that the follow-up group might have smaller mean but larger SD To accommodate this situation; we have modified the Sarkadi's method as described in online supplementary technical appendix and online supplementary figure 1 . The health gain distributions are presented in online supplementary figure 2 to illustrate the health gains at a population level.

In addition to the normal distribution originally used in Sarkadi's method, three other distributions, gamma distribution, log-normal distribution and normal distribution of log-transferred payment data, were attempted to fit the data. The goodness-of-fit statistics, Akaike information criterion (AIC), Bayesian information criterion (BIC) and log-likelihood were tested over four distributions and the distribution with the minimum AIC, BIC and maximum log-likelihood was chosen as the final distribution to examine the impact.

Bootstrapping is used to obtain a $\mathrm{P}$ value for the probability of health gain larger than zero. We randomly sampled data points with replacements from the original data separately for the baseline and follow-up, so that we obtain bootstrapped data with the same numbers of data points. These are used to obtain an estimation of the health gain after perturbation. This process is repeated 1000 times. The probability of observing estimations less than or equal to zero is calculated, and used as the approximation of the $\mathrm{P}$ value for testing whether health gain is significantly larger than zero.

No personal identifiers were released to researchers, and all subsequent analyses were conducted on anonymised datasets. Age data were provided allowing analyses to be undertaken above and below the median age (70 years) to assess any related variation.

The work had approval from the Cambridgeshire research ethics committee as part of a wider service evaluation and, as such, was deemed not to require personal informed consent.

All analyses were conducted in R (V.3.1).

\section{RESULTS}

The sample size of inpatient payment records during the baseline and the intervention periods in each region is presented in table 1 . The inpatient payments during the baseline period and the intervention period are shown in table 2 by area and age group. In each area and age group, a lower individual median inpatient payment was more likely to be found in the intervention period.

Figure 1 shows the distribution of the inpatient payments in people with type 2 diabetes in the baseline and intervention periods. This illustrates the effect of the integrated care intervention, as the left-moving curve in the intervention period indicates the potential inpatient payment saving at a population level.

Four distributions (normal distribution, gamma distribution, log-normal distribution and normal 
Table 1 Sample size of the inpatient payment records

\begin{tabular}{|c|c|c|c|c|c|c|}
\hline & \multicolumn{2}{|c|}{ East Cambridge and Fenland } & \multicolumn{2}{|c|}{ Huntingdonshire } & \multicolumn{2}{|c|}{ Great Cambridge } \\
\hline & $<70$ years & $\geq 70$ years & $<70$ years & $\geq 70$ years & $<70$ years & $\geq 70$ years \\
\hline 2008-2009 & 2012 & 2028 & 1494 & 1664 & 1575 & 1329 \\
\hline
\end{tabular}

distribution of log-transformed payment data) were attempted to fit the payment data as presented in online supplementary table 1 . The normal distribution of log-transformed payment data was chosen to estimate the impact on the intervention for its minimum AIC and BIC and its maximum log-likelihood.

The magnitude of the intervention at the population level is presented in table 3. Significant 'health gain' was observed both in the intervention area and control areas, especially among patients aged less than 70 years. In the intervention area, East Cambridge and Fenland, $7.69 \%$ (95 CI $5.89 \%$ to $9.74 \%$ ) and $2.05 \%(0.72 \%$ to $4.13 \%$ ) of patients aged less than 70 years and aged more than 70 years, respectively, had a reduced inpatient payment, compared with the population in the baseline period. In Huntingdonshire, the 'health gain' was $6.90 \%$ (5.63\% to $8.68 \%)$ and $4.62 \%(2.22 \%$ to $7.23 \%)$ among patients aged less than 70 years and patients aged more than 70 years, respectively. In Greater Cambridge, the 'health gain' was $7.59 \%(5.63 \%$ to $9.94 \%)$ and $2.49 \%$ $(1.46 \%$ to $4.58 \%)$ among patients aged less than 70 years and patients aged more than 70 years, respectively.

To allow comparisons, the estimated impact, based on a normal distribution, is presented in online supplementary table 2. In the intervention area, East Cambridge and Fenland, 2.74\% (1.29\% to $5.81 \%$ ) of patients aged more than 70 years had a reduced inpatient payment, compared with the population in the baseline period. In one of the control areas, Greater Cambridge, 'health gain' was also observed in $3.20 \%$ ( $1.77 \%$ to 7.20$)$ of patients aged less than 70 years and $4.14 \%(2.27 \%$ to 7.86$)$ patients aged more than 70 years, respectively. Significant 'health gain' was not identified within the population in Huntingdonshire over the study period.

\section{DISCUSSION}

We have used a novel way, calculating the total health gain (proportion of people with reduced inpatient payments) assuming a Gaussian distribution, to assess the results of integrated care in the diabetic population of areas in Cambridgeshire through a population lens. The study revealed a possible effect of the new integrated care approach on inpatient payments, as $7.7 \%$ of patients aged less than 70 years and $2.1 \%$ of patients aged more than 70 years had reduced inpatient payments in the intervention area, East Cambridge and Fenland. However, reductions were also seen in the control areas, in Huntingdonshire, $6.9 \%$ of patients aged less than 70 years and $4.6 \%$ of patients aged less than 70 years had reduced inpatient payment; in Greater Cambridge, $7.6 \%$ of patients aged less than 70 years and $2.5 \%$ of patients aged less than 70 years had reduced inpatient. The 95\% CIs overlapped across the three areas, so we have not shown any differences between the areas.

Significant improvements in diabetes care can occur with multifaceted interventions ${ }^{16}$ including disease management in the $\mathrm{USA}^{17}$ and integrated care in Germany, ${ }^{18}$ and these can be associated with reductions in hospital costs. ${ }^{19}$ The integrated care intervention was successfully implemented across the area, with positive patient experience, improved practice nurse clinical confidence and early reports of clinical benefit. ${ }^{13} 20$ It is therefore surprising that although some (small) positive benefit was observed in the intervention area, the return on the investment of $£ 250000$ was not greater and possibly less than in one of the control areas. Elsewhere, diabetes integrated care interventions have generally been more effective within single providers or in contexts where multiple primary care organisations work with a single specialist provider under an integrated insurance scheme. ${ }^{6}$ The integrated care intervention carried out in ECF followed a nurse-led service with one of the goals reducing referrals (ie, payments) to hospital outpatients. This philosophy, rather than progressing to truly integrated services, carried through the intervention period, although as part of a wider programme that included 'vertical integration' developments. It was perhaps to be expected that attempts at creating such greater 'vertical' integration in information management, clinical governance, budget and overall management were agreed, but not implemented, actions more achievable within a single organisation. There was an attempt to create a single equal partner network model ${ }^{21}$ nearing the end of the intervention period, but this was not funded by the local commissioners.

The failure to implement integrated information management almost certainly contributed to communication and integration difficulties. Most integrated care initiatives attempt to include data sharing ${ }^{22}$ and this was not possible within the local information governance arrangements. This was noticed by the patients and was a source of frustration. Interestingly, integration was perceived as happening when there was one person 'fronting up' for all those involved. Case management has been proposed as one approach to integration, and requires the case manager to corral and coordinate the services for a given individual. ${ }^{17}$ 
Whether our findings are due to a unique set of circumstances or expected as part of a three-compartment model (primary care, intermediate care and secondary care) is unclear, but there are indications that the circumstances are not special. There are calls for more integration and less fragmentation in healthcare, ${ }^{22}$ yet the evidence on what works in England is limited. ${ }^{23}{ }^{24}$ The latest changes in commissioning in the English NHS, with emphasis on the need to consider 'Any qualified Provider' in service delivery, and associated market procurement approaches, could well impair the quality of diabetes care while increasing overall cost, if the experience here is reproduced elsewhere.

Similarly, as a 'natural experiment', it was not possible to measure the impact of integrated care on inpatient payments at an individual level. Instead, we estimated the proportion of the population showing 'health gain' (reduced inpatient payments) from the integrated care intervention by using the distribution curve of inpatient payment. Although the method was within the conceptual framework proposed by Sarkardi, some modifications to the methodology were made to overcome methodological drawbacks, for example, requiring the same SD for two Gaussian curves: something unlikely to occur in real scenarios. We believe this revised method would be more applicable to evaluate the 'health gain' for interventions at a population level.

There are limitations to our study. This was not a randomised trial, so any changes could be due to secular trends, although we do compare with the two other areas in Cambridgeshire. The data depended on the completeness of the coding of diabetes, and there being no systematic change in coding over this time period. We found that at least one provider had high diabetes ascertainment. ${ }^{10}$ Data access restrictions prevented adjustment for some important covariables. As the data used was record based rather than individual based, repeat inpatient records were unable to be linked; however, the record-based data still provide a range of plausible estimations. Moreover, within a relatively fixed diabetes population served by a local 'closed' inpatient care and tariff system, the likelihood for patients having a second hospital admission would still be relatively low (although higher than those without diabetes).${ }^{10}$ In other words, inpatient payments at two time points are considered completely independent of each other. We acknowledge that this current analysis still yields findings subject to confounding bias unable to be measured in this study. The 'impact' observed in our study may therefore only reflect measured changes in the DICI and 'control' regions, respectively, rather than due to the DICI itself, as the DICI care model was not randomly assigned. As a result of data access restrictions, it is not possible in this study to identify those with multiple admissions (and payments) that would provide 'redundant information'. The application of bootstrapping ignoring such redundant information might lead to a misapplication of Sarkadi's tool and might inadvertently increase the false-positive rate: something to be 

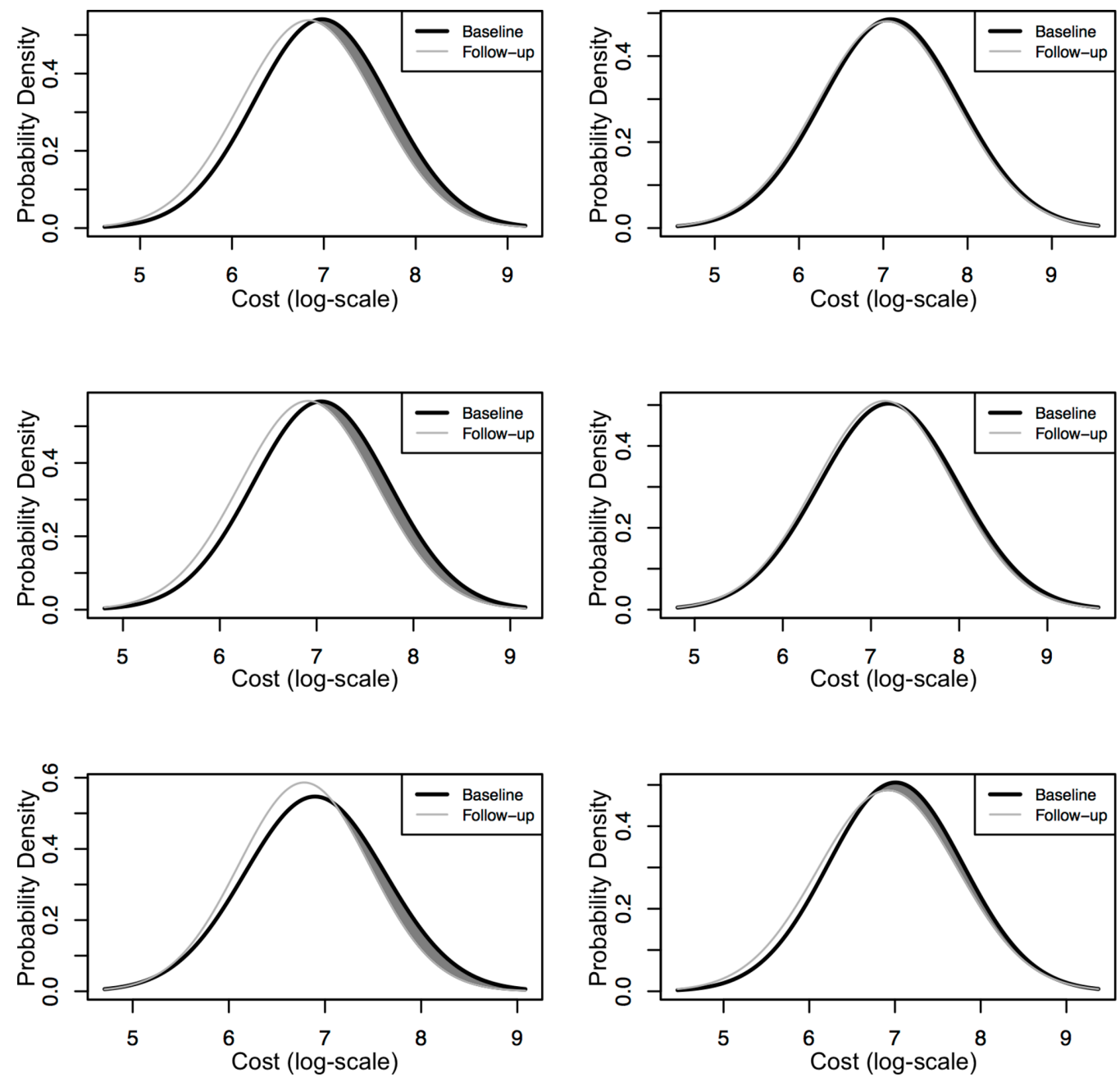

Figure 1 Using the normal (Gaussian) curve to demonstrate the distribution of inpatient payment in people with type 2 diabetes and possible effects of an integrated care on the curve. The differences between the respective areas under the curve are shaded. Health gains for participants with lower inpatient payment. Left top: East Cambridge and Fenland, $<70$ years; right top: East Cambridge and Fenland, $\geq 70$ years. Left middle: Great Cambridge, $<70$ years; right middle: Great Cambridge, $\geq 70$ years. Left bottom: Huntingdonshire, $<70$ years; right bottom: Huntingdonshire, $\geq 70$ years.

taken into consideration when interpreting the findings in this study. There might be other potential unidentified confounders in this study and evaluation seeking other confounding factors would be possible in future studies with more variables in the dataset including a way to identify those confounders.
In conclusion, we have applied a modified novel strategy to measure 'health gain' associated with an integrated care intervention at a population level. We found that there were no differences in inpatient payments. Our findings suggest that irrespective of the ideal principles behind integration, linking multiple health providers to

Table 3 The estimated absolute 'health gain (impact)' after the intervention by age and region: estimation based on normal distribution of log-transferred inpatient payment data

\begin{tabular}{lllll}
\hline & & Impact, \% & $\mathbf{9 5 \%} \mathbf{C l}, \%$ & P value (bootstrapping) \\
\hline East Cambridge and Fenland & $<70$ years & 7.69 & $(5.89$ to 9.74$)$ & 0 \\
& $\geq 70$ years & 2.05 & $(0.72$ to 4.13$)$ & 0.044796 \\
Huntingdonshire & $<70$ years & 6.90 & $(5.63$ to 8.68$)$ & 0 \\
& $\geq 70$ years & 4.62 & $(2.22$ to 7.23$)$ & 0.001300 \\
Greater Cambridge & $<70$ years & 7.59 & $(5.63$ to 9.94$)$ & 0 \\
& $\geq 70$ years & 2.49 & $(1.46$ to 4.58$)$ & 0.037096
\end{tabular}

The health gain (impact) was defined as percentage of people with type 2 diabetes and hospital admission having reduced inpatient payment after the integrated care at population level. 
deliver population-based diabetes care is complex and improvements in health outcomes remain difficult to achieve.

\section{Author affiliations}

${ }^{1}$ Department of Nephrology, The First Affiliated Hospital, Zhengzhou University, Zhengzhou, China

${ }^{2}$ Arthritis Research UK Primary Care Centre, Research Institute for Primary Care and Health Sciences, Keele University, Keele, UK

${ }^{3}$ School of Medicine, Washington University in St Louis, St Louis, Missouri, USA

${ }^{4}$ Macarthur Clinical School, Western Sydney University, Sydney, Australia

Contributors DY analysed the data and drafted the manuscript; WY revised the statistical methods and revised the manuscript; $Y C$ validated the method and reanalysed the data independently; ZZ designed the analysis framework and revised the manuscript; DS designed the study, revised the analysis framework, revised the manuscript and interpreted the findings.

Funding This paper presents independent research funded by the National Institute for Health Research (NIHR) under its Research for Patient Benefit (RfPB) Programme (Grant Reference Number PB-PG-0808-17303).

Disclaimer The views expressed are those of the authors and not necessarily those of the NHS, the NIHR or the Department of Health.

Competing interests None declared.

Ethics approval National Research Ethics Service Committee-East of England.

Provenance and peer review Not commissioned; externally peer reviewed.

Data sharing statement № additional data are available.

Open Access This is an Open Access article distributed in accordance with the Creative Commons Attribution Non Commercial (CC BY-NC 4.0) license, which permits others to distribute, remix, adapt, build upon this work non-commercially, and license their derivative works on different terms, provided the original work is properly cited and the use is non-commercial. See: http://creativecommons.org/ licenses/by-nc/4.0/

(c) Article author(s) (or their employer(s) unless otherwise stated in the text of the article) 2017. All rights reserved. No commercial use is permitted unless otherwise expressly granted.

\section{REFERENCES}

1. Kar P. The 'super six' for the acute trust; all else under primary care? Practical Diabetes 2011;28:308-9.

2. Nagi D, Wilson J. Integrated diabetes care: the Wakefield diabetes service redesign. Practical Diabetes 2011;28:310-1.

3. Rea RD, Gregory S, Browne M, et al. Integrated diabetes care in Derby: new NHS organisations for new NHS challenges. Practical Diabetes 2011;28:312-3.

4. Panos K, Svd A, Schurer W. Diabetes expenditure, burden ofdisease and management in 5 EUcountries. London, UK: The London School of Economics and Political Science, 2012.

5. Arts EE, Landewe-Cleuren SA, Schaper NC, et al. The costeffectiveness of substituting physicians with diabetes nurse specialists: a randomized controlled trial with 2-year follow-up. J Adv Nurs 2012;68:1224-34.

6. Simmons D, Yu D, Bunn C, et al. Hospitalisation among patients with diabetes associated with a Diabetes Integrated Care Initiative: a mixed methods case study. Future Hosp J 2015;2:92-8.

7. Wenzel H, Simmons D. Chapter-1: An introduction to integrated care and diabetes integrated care. integrated diabetes care: a multidisciplinary approach. Springer 2016.

8. Wilson A, O'Hare JP, Hardy A, et al. Evaluation of the clinical and cost effectiveness of intermediate care clinics for diabetes (ICCD): a multicentre cluster randomised controlled trial. PLoS One 2014:9:e93964

9. Sarkadi A, Sampaio F, Kelly MP, et al. A novel approach used outcome distribution curves to estimate the population-level impact of a public health intervention. J Clin Epidemiol 2014;67:785-92.

10. Simmons D, Wenzel H. Diabetes inpatients: a case of lose, lose, lose. Is it time to use a 'diabetes-attributable hospitalization cost' to assess the impact of diabetes? Diabet Med 2011:28:1123-30.

11. Simmons $D, Y u D$, Wenzel $H$. Changes in hospital admissions and inpatient tariff associated with a diabetes integrated care initiative: preliminary findings. J Diabetes 2014;6:81-9.

12. Hollern $\mathrm{H}$, Lunn J. Development of a primary care specialist diabetes nursing service. J Diabetes Nursing 2004;8:344-8.

13. Harwood E, Bunn C, Caton C, et al. Addressing barriers to diabetes care and self-care in general practice: a new framework for practice nurses. J Diab Nursing 2013;17:186-91.

14. Park SK, Wang W. Ambient air pollution and type 2 diabetes: a systematic review of epidemiologic research. Curr Environ Health Rep 2014;1:275-86.

15. Wild SH, McKnight JA, McConnachie A, et al. Glasgow and Lothian Diabetes Register Data Group.Socioeconomic status and diabetes-related hospital admissions: a cross-sectional study of people with diagnosed diabetes. J Epidemiol Community Health 2010;64:1022-4.

16. Renders CM, Valk GD, Griffin SJ, et al. Interventions to improve the management of diabetes in primary care, outpatient, and community settings: a systematic review. Diabetes Care 2001;24:1821-33.

17. Norris SL, Nichols PJ, Caspersen CJ, et al. The effectiveness of disease and case management for people with diabetes. a systematic review. Am J Prev Med 2002;22:15-38.

18. Rothe U, Muller G, Schwartz PEH, et al. Evaluation of a diabetes management system based on practice guidelines, integrated care, and continuous quality management in a federal state of germany: a population-based approach to health care research. Diabetes Care 2002;25:684-9.

19. Sidorov J, Shull R, Girolami S, et al. Does diabetes disease management save money and improve outcomes? Diabetes Care 2002;25:684-68.

20. Hollern H, Simmons D. Cost saving and improved glycaemic control in an integrated diabetes service. Primary Care Diabetes 2011;13:176-81.

21. Simmons D, Wenzel H, Zgibor JC. Chapter-15: diabetes integrated care: are we there yet? integrated diabetes care: a multidisciplinary approach. Springer 2016.

22. Ham C, Walsh N. Making integrated care happen at scale and pace. London: Kings Fund, 2013.

23. Ham C, Curry N. Integrated care: What is it? Does it work? What does it mean for the NHS? London: Kings fund, 2011.

24. RAND Europe, Ernst \& Young LLP. National Evaluation of the Department of Health's Integrated Care Pilots: final report:full version. RAND Europe, Ernst \& Young LLP London. 2012. 